\title{
Guanfacine Hydrochloride
}

National Cancer Institute

\section{Source}

National Cancer Institute. Guanfacine Hydrochloride. NCI Thesaurus. Code C47550.

The hydrochloride salt form of guanfacine, a centrally acting alpha-2 adrenergic agonist with antihypertensive activity. Alpha-2 receptor stimulation by guanfacine hydrochloride results in a decreased sympathetic outflow from the vasomotor center to the heart, kidneys, and peripheral vasculature. This leads to a decrease in heart rate and peripheral vascular resistance. ( $\mathrm{NCl05)}$ 